\title{
SIGNIFICANCE OF LOG-PERIODIC SIGNATURES IN CUMULATIVE NOISE
}

\author{
HANS-CHRISTIAN GRAF V. BOTHMER
}

\begin{abstract}
Using methods introduced by Scargle we derive a cumulative version of the Lomb periodogram that exhibits frequency independent statistics when applied to cumulative noise. We show how this cumulative Lomb periodogram allows us to estimate the significance of log-periodic signatures in the S\&P 500 anti-bubble that started in August 2000 .
\end{abstract}

\section{INTRODUCTION}

Speculative bubbles, crashes and depressions are some of the most puzzling phenomena in financial markets. Even though these are rare events they occur much more often, than expected from standard models of financial markets. For example the otherwise very successfull GARCH-Model predicts only one crash of a magnitute comparable to the events of 1929 and 1987 in 16000 years JS98. This suggest that the very largest crashes are outliers that are caused by phenomena not considered in the standard models.

Several authors DS96, JAF96, VBMA98, have argued that this behavior is natural, if one considers the stock-market as a complex system. When this system is far away from critical points, standard models are a good description of the systems dynamics, but when a critical point is approached the dynamics change completely. In this picture a crash occurs, when a critical point is reached.

The theory of critical phenomena predicts that all observables of a complex system near a critical point are scale invariant. In the case of stock markets this would mean that the price $p(t)$ near a critical point should follow a power-law

$$
\log p(t) \sim A+B\left(t_{c}-t\right)^{\beta} .
$$

where $t_{c}$ is the time of crash. Unfortunately it is rather difficult to distinguish this from the exponential growth

$$
\log p(t) \sim A+B t
$$

predicted by standard theory.

Help in this situation comes from the concept of discrete scale invariance. If the system considered has a hirachical structure it might scale only in accordance with these hirachies. This allows the scaling coefficient $\beta$ to be

Date: November 6, 2018. 
a complex number and the observables will obey

$$
\begin{aligned}
\log p(t) \sim & \operatorname{Re}\left(A+B\left(t_{c}-t\right)^{\beta}\right) \\
& =A+B\left(t_{c}-t\right)^{\alpha}+C\left(t_{c}-t\right)^{\alpha} \cos \left(\omega \ln \left(t_{c}-t\right)+\phi\right),
\end{aligned}
$$

Such a power-law with log-periodic signature is much easier to detect than a pure power-law. In fact sizable log-periodic signatures have been detected before many financial crashes, including the ones from 1929 and 1987 ([DS96], JAF96], VBMA98], SLJ99], [AJ00]). Also they have been found after the bursting of some speculative bubbles, for example gold after 1980, the Nikkei-Index after 1990 and most recently after the bursting of the new economy bubble in 2000 ( AJ99, [SZ02]).

Still there is some reason for doubt. As several authors have pointed out $\left(\mathrm{HJL}^{+} 00\right.$, Fei01b] $)$, log-periodic signatures can often arise by chance in systems that do not exhibit discrete scale invariance. Even simple Brownian motion produces log-periodic signatures quite often. Therefore there has been a heated discussion about the significance of the found log-periodic signatures in financial data Fei01b, SJ01, Fei01a.

One tool to detect these signatures has been the Lomb periodogram introduced by Lomb [Lom76] and improved by Scargle in [Sca82. An important property of Scargle's periodogram is that the individual Lomb powers of independently normal distributed noise follow approximately an exponential distribution.

Unfortunately this property is lost, when one calculates Scargle's periodogram for cumulative noise, where the differences between two observations are independently normal distributed. In this Brownian-Motion case, the expected Lomb powers are much greater for small frequencies than for large ones. Therefore the significance of large Lomb powers at small frequencies is difficult to estimate. Huang, Saleur, Sornette and Zhou tackle this problem and several related ones with extensive Monte Carlo simulations in $\mathrm{HJL}^{+} 00$ and $\mathrm{ZS02}$.

In this paper we present an analytic approach to the problem of estimating the significance of log-periodic signatures. We start by introducing a small correction to Scargle's Lomb periodogram, that makes the distribution of Lomb powers exactly exponential for independently normal distributed noise.

In the second part we use the same methods to derive a normalisation of the Lomb periodogram that assures an frequency independent exponential distribution of Lomb powers for cumulative noise.

In the last section we apply these new methods to estimate the significance of log-periodic signatures in so called S\&P 500-anti-bubble after the crash of 2000. We show how our methods greatly simplify the whole analysis and derive that there is about a $6 \%$ chance that a signature like the one detected by Sornette and Zhou in [SZ02 arises by chance if one only considers frequencies smaller than 10.0. If one searches all frequencies up to the Nyquist frequency, peaks of this height become much more common.

Furthermore we detect equally significant peaks at harmonics of the fundamental frequency of Sornette and Zhou. This complements evidence for a 
more sophisticated modelling of the S\&P 500 anti-bubble by Sornette and Zhou in ZS03.

We would like to thank Volker Möller for hosting our database of financial data and donating cpu-time for the Monte Carlo simulations that lead to this paper.

\section{INDEPENDENT NOISE}

Consider the classical periodogram

$$
P(\omega)=\frac{1}{N_{0}}\left[\left(\sum_{j=1}^{N_{0}} X_{j} \cos \omega t_{j}\right)^{2}+\left(\sum_{j=1}^{N_{0}} X_{j} \sin \omega t_{j}\right)^{2}\right] .
$$

This is a generalization of the Fourier transform to the case of unevenly spaced measurements. Unfortunately this classical form has difficult statistical behavior for uneven spacing. Scargle therefore proposed in Sca82 a normalized from of the periodogram, that restores good statistical properties in the case where all $X_{j}$ are independently normal distributed with mean zero and variance $\sigma_{0}^{2}$. For this he observes that

$$
S(w)=\sum_{j=1}^{N_{0}} X_{j} \cos \omega t_{j}
$$

and

$$
C(w)=\sum_{j=1}^{N_{0}} X_{j} \sin \omega t_{j}
$$

are again normally distributed, with variances

$$
\sigma_{c}^{2}=N_{0} \sigma_{0}^{2} \sum_{j=1}^{N_{0}} \cos ^{2} \omega t_{j}
$$

and

$$
\sigma_{s}^{2}=N_{0} \sigma_{0}^{2} \sum_{j=1}^{N_{0}} \sin ^{2} \omega t_{j} .
$$

Also he claims that for normally distributed random variables with unit variance their sum of squares is exponentially distributed. Therefore he proposes to look at

$$
P(\omega)=\left[\frac{\left(\sum_{j=1}^{N_{0}} X_{j} \cos \omega t_{j}\right)^{2}}{\sigma_{c}}+\frac{\left(\sum_{j=1}^{N_{0}} X_{j} \sin \omega t_{j}\right)^{2}}{\sigma_{s}}\right] .
$$

Scargles claim, that this sum is exponentially distributed is only correct when $S(\omega)$ and $C(\omega)$ are independent. This is approximately true, when the observation times $t_{j}$ are "not to badly bunched". In other cases whole variance/covariance matrix

$$
\Sigma=\left(\begin{array}{cc}
\sigma_{c}^{2} & \sigma_{c s} \\
\sigma_{c s} & \sigma_{s}^{2}
\end{array}\right)
$$


with

$$
\sigma_{c s}=N_{0} \sigma_{0}^{2} \sum_{j=1}^{N_{0}} \cos \omega t_{j} \sin \omega t_{j}
$$

has to be considered.

If $\Sigma$ is invertible the values of the quadratic form

$$
P(\omega)=\frac{1}{2}(C(\omega), S(\omega)) \Sigma^{-1}\left(\begin{array}{c}
C(\omega) \\
S(\omega)
\end{array}\right)
$$

are exponentially distributed, i.e.

$$
\operatorname{Probability}(P(\omega)>z)=\exp (-z) .
$$

by standard facts about multivariate normal distributions (for example Mui82, Theorem 1.4.1]). Explicitly we can calculate

$$
\Sigma^{-1}=\frac{1}{\sigma_{c}^{2} \sigma_{s}^{2}-\sigma_{c s}^{2}}\left(\begin{array}{cc}
\sigma_{s}^{2} & -\sigma_{c s} \\
-\sigma_{c s} & \sigma_{c}^{2}
\end{array}\right) .
$$

This means the natural form of the periodogram is

$$
P(\omega)=\frac{1}{2} \frac{\sigma_{s}^{2} C^{2}(\omega)+\sigma_{c}^{2} S^{2}(\omega)-2 \sigma_{c s} C(\omega) S(\omega)}{\sigma_{c}^{2} \sigma_{s}^{2}-\sigma_{c s}^{2}},
$$

which reduces to Scargle's case for $C$ and $S$ independent $\left(\sigma_{c s}=0\right)$, and to the classical case for even spacing $\left(\sigma_{c}=\sigma_{s}\right)$. This $P(\omega)$ is always exponentially distributed.

As Scargle we also replace $t_{j}$ by $t_{j}-\tau$ with

$$
\tau=\frac{1}{2 \omega} \arctan \frac{\sum_{j=1}^{N_{0}} \sin 2 \omega t_{j}}{\sum_{j=1}^{N_{0}} \cos 2 \omega t_{j}}
$$

in all formulas, to make the diagram time-translation invariant.

\section{Cumulative Noise}

Assume now, that the differences $Y_{j}=\left(X_{j}-X_{j-1}\right)$ are independently normal distributed with zero mean and variance $\sigma_{0}^{2}$. Then the distribution of powers in Scargle's periodogram depends on the angular frequency $\omega=2 \pi f$. See figure 1 for the result of a Monte Carlo simulation of this case. In small frequencies much higher Lomb powers can occur by chance, then in high frequencies. This reflects the well known fact that the expected value at frequency $f$ of the evenly spaced Fourier transform of a brownian motion is proportional to $1 / f^{2}$. Our idea is now to normalize the periodogram by adjusting Scargle's methods to this case.

First notice that

$$
X_{j}-X_{0}=\sum_{k=1}^{j} Y_{k}
$$


in this situation. From this we obtain

$$
\begin{aligned}
C(\omega) & =\sum_{j=1}^{n}\left(X_{j}-X_{0}\right) \cos \omega t_{j} \\
& =\sum_{j=1}^{n}\left(\sum_{k=1}^{j} Y_{k}\right) \cos \omega t_{j} \\
& =\sum_{k=1}^{n} Y_{k} \sum_{j=k}^{n} \cos \omega t_{j}
\end{aligned}
$$

and a similar formula for $S(\omega)$. Notice that $C(\omega)$ and $S(\omega)$ are still normally distributed with

$$
\begin{aligned}
\sigma_{c}^{2} & =\left\langle C^{2}(\omega)\right\rangle \\
& =\sum_{k l}\left\langle Y_{k}, Y_{l}\right\rangle\left(\sum_{j=k}^{n} \cos \omega t_{j}\right)\left(\sum_{j=l}^{n} \cos \omega t_{j}\right) \\
& =\sigma_{0}^{2} \sum_{k=1}^{n}\left(\sum_{j=k}^{n} \cos \omega t_{j}\right)^{2}
\end{aligned}
$$

and

$$
\begin{aligned}
\sigma_{s}^{2} & =\left\langle S^{2}(\omega)\right\rangle \\
& =\sum_{k l}\left\langle Y_{k}, Y_{l}\right\rangle\left(\sum_{j=k}^{n} \sin \omega t_{j}\right)\left(\sum_{j=l}^{n} \sin \omega t_{j}\right) \\
& =\sigma_{0}^{2} \sum_{k=1}^{n}\left(\sum_{j=k}^{n} \sin \omega t_{j}\right)^{2}
\end{aligned}
$$

and

$$
\begin{aligned}
\sigma_{c s} & =\langle C(\omega) S(\omega)\rangle \\
& =\sum_{k l}\left\langle Y_{k}, Y_{l}\right\rangle\left(\sum_{j=k}^{n} \sin \omega t_{j}\right)\left(\sum_{j=l}^{n} \cos \omega t_{j}\right) \\
& =\sigma_{0}^{2} \sum_{k=1}^{n}\left(\sum_{j=k}^{n} \cos \omega t_{j}\right)\left(\sum_{j=k}^{n} \sin \omega t_{j}\right) .
\end{aligned}
$$

With these values

$$
P(\omega)=\frac{1}{2} \frac{\sigma_{s}^{2} C^{2}(\omega)+\sigma_{c}^{2} S^{2}(\omega)-2 \sigma_{c s} C(\omega) S(\omega)}{\sigma_{c}^{2} \sigma_{s}^{2}-\sigma_{c s}^{2}},
$$

is again exponentially distributed:

$$
\operatorname{Probability~}(P(\omega)>z)=\exp (-z)
$$

In particular the distribution is now independent of $\omega$ as exemplified by figure 2 which shows the cumulative Lomb periodogram for a Monte Carlo simulation as above.

Notice that is essential to consider the correlation between $C(\omega)$ and $S(\omega)$ in this case. Figure 3 shows the cumulative Lomb periodograms for 1000 
random walks of length 500 in logarithmic time without the correlation term above. Notice how this introduces spurious peaks at several frequencies.

Again we replace $t_{j}$ by $t_{j}-\tau$ with

$$
\tau=\frac{1}{2 \omega} \arctan \frac{\sum_{j=1}^{N_{0}} \sin 2 \omega t_{j}}{\sum_{j=1}^{N_{0}} \cos 2 \omega t_{j}}
$$

in all formulas, to make the diagram time-translation invariant.

\section{Application to LOG-PERIOdicity in FinAnCial DATA}

Recently Sornette and Zhou have suggested that there is a log periodic signature in the S\&P 500 index after the bursting of the new economy bubble SZ02. Among other methods they use Scargle's periodogram with $\tilde{t}_{j}=$ $\log \left(t_{j}-t_{c}\right)$ and $X_{j}$ the logarithm of the index price $j$ days after a critical date $t_{c}$ for frequencies $0 \leq f \leq 10$. To account for a nonlinear trend of the form

$$
A+B\left(t_{j}-t_{c}\right)^{\alpha}
$$

they first detrend the index values. For this they use a value of $A$ obtained from a nonlinear fit of

$$
(*) \quad A+B\left(t_{j}-t_{c}\right)^{\alpha}+C\left(t_{j}-t_{c}\right)^{\alpha} \cos \left(\omega \ln \left(t_{j}-t_{c}\right)+\phi\right)
$$

to the logarithm of the index price and then determine $B$ and $\alpha$ for different choices of $t_{c}$ by linear regression.

We follow the same procedure, but estimate $A$ by a different method. For this we consider the correlation coefficient

$$
r_{A}=\frac{\sum_{i}\left(\tilde{t}_{j}-\bar{t}\right)\left(\log \left(X_{j}-A\right)-\bar{X}\right)}{\sqrt{\sum_{i}\left(\tilde{t}_{j}-\bar{t}\right)^{2}} \sqrt{\sum_{i}\left(\log \left(X_{j}-A\right)-\bar{X}\right)^{2}}}
$$

with $\bar{t}$ the average of the $\tilde{t_{j}}$ 's and $\bar{X}$ the average of the $\log \left(X_{j}-A\right)$ 's. This correlation coefficient measures how well $\log \left(X_{j}-A\right)$ can be approximated by a linear model

$$
\log B+\alpha \log \left(t_{j}-t_{c}\right) .
$$

Notice that $r_{A}$ does not depend on $B$ and $\alpha$. Since correlation coefficiens near +1 and -1 indicate strong explanatory value of the proposed trend, while coefficients near 0 indicate that a trend with this value of $A$ seems not to be present, we first search the value of $A$ that maximises $r_{A}^{2}$. Then, like Sornette and Zhou, we find $B$ and $\alpha$ by linear regression

With this method we address a critique of Feigenbaum [Fei01a. He points out that the detection of log-periodicity by lomb periodograms is not independent of the detection via nonlinear fits of equation $(*)$ to the price data, if values produced by the second are reused in the first. Our approach eliminates this dependance.

Figure 4 shows the highest Lomb powers obtained by this method for 2year intervals starting from August 1st to September 5th, 2000. The highest peak in our dataset is observed for the critical date August 22th, 2000. This is in reasonable agreement with the critical date found by Sornette and Zhou (August 9th, 2000). 
To estimate the significance of this peak we calculated the 203 Lomb periodograms of 2-year intervals starting at the 22nd of each month from Jannuary 1984 to November 2000. Figure [5 shows a comparison of these periodograms with the one from August 22nd, 2002. Notice that the distribution of Lomb-powers depends strongly on the frequency. For small frequencies high Lomb powers are much more probable than at high frequencies. The absence of large powers for very small frequencies is due to the detrending procedure. These facts have also been observed by $\mathrm{HJL}^{+} 00$ in a somewhat different setting.

Notice also that the peak at $f \approx 1.6$ is nevertheless quite large, in fact it is the largest one observed at this frequency. But how probable is it that we have a peak of this relative height for any of the tested frequencies? The SnP500 data set is not large enough, to estimate this probability accurately, but a count shows that there are 39 datasets that have at least one frequency with a peak that is higher than any of the other at the same frequency. This gives a naive estimate of $39 / 202 \approx 19,3 \%$.

The cumulative Lomb periodogram proposed above improves and greatly simplifies this analysis. Figure [6] shows the cumulative Lomb periodograms of the S\&P 500 anti-bubble together with the cumulative Lomb periodograms of all other datasets. A detrending of the data as above was not necessary. Notice that there are now several peaks of comparable size at frequencies 1.7, 3.4, 7.4 and 8.4. The fact that these lie close to the harmonics of 1.7 compares well to the results of [ZS03].

We have included the theoretical 99.9\%, 99\% and 95\%-quantiles for each frequency derived above together with the 95\%-quantiles of the actual S\&P 500 data. Notice the excellent agreement for frequencies greater than 1.

Since the height of the peaks is now largely independent from the frequency, we can estimate the global significance of the peak at 1.7 with cumulative Lomb power 5.61 by counting the number of cumulative Lombperiodograms with at least one peak of higher power for frequencies $0 \leq$ $f \leq 10$. We find 12 of those which implies a significance of approximately $12 / 203 \approx 5.9 \%$. In figure 7 we compare the global significance of peaks in cumulative Lomb periodograms of the S\&P 500 with those obtained from a Monto Carlo simulation of brownian motion with zero mean and unit variance. There is a reasonable agreement for significances smaller than 0.1 even though brownian motion is only a very rough model of stock market prices.

Notice that the global significance of a peak depends strongly on the number of frequencies considered. If we consider all frequencies up to the Nyquist frequency for the average sampling interval

$$
f_{N}=\frac{N_{0}}{2 T}=\frac{500}{2 \log (500)} \approx 40.2
$$

a peak of height 5.61 as above is found in $27.6 \%$ of simulated periodograms and in $28.3 \%$ of the historical S\&P 500 periodograms. In this light the choice of $0 \leq f \leq 10$ by Sornette and Zhou seems to call for an apriori justification if one wants to keep up the claim of a significant log-periodic signature around $f=1.7$. 


\section{Conclusion}

We have indrocuced a new version of the Lomb periodigram that exhibits good statistical properties when applied to cumulative noise. With this we were able to detect the log-periodic signature in the S\&P 500 anti-bubble with better significance than with the ususal periodigram, even without a detrending procedure. More importantly this method allows us to estimate the significance of the found log periodic signature which is a reasonable $5.9 \%$ if we only consider fequencies smaller than 10.0, and a disappointing $27.6 \%$ if one considers all frequencies up to the Nyquist frequency for the average sampling interval. We also detect cumulative Lomb peaks of similar significance at harmonics of the fundamental frequency 1.7.

\section{REFERENCES}

[AJ99] Didier Sornette Anders Johansen. Financial "anti-bubbles": Log-periodicity in gold and nikkei collapses. International Journal of Modern Physics C, 10(4):563-575, 1999.

[AJ00] Didier Sornette Anders Johansen. The nasdaq crash of april 2000: Yet another example of log-periodicity in a speculative bubble ending in a crash. European Physical Journal B, 17:319-328, 2000.

[DS96] Jean-Philippe Bouchaud Didier Sornette, Anders Johansen. Stock market crashes, precursors and replicas. J.Phys.I France, 6:167-175, 1996.

[Fei01a] James A. Feigenbaum. More on a statistical analysis of log-periodic precursors to financial crashes. Quantitative Finance, 1(5):527-532, 2001.

[Fei01b] James A. Feigenbaum. A statistical analysis of log-periodic precursors to financial crashes. Quantitative Finance, 1(3):346-360, 2001.

$\left[\mathrm{HJL}^{+} 00\right]$ Y. Huang, A. Johansen, M. W. Lee, H. Saleur, and D. Sornette. Artifactual log-periodicity in finite-size data: Relevance for earthquake aftershocks. $J$. Geophysical Research, 105(B12):28111-28123, 2000.

[JAF96] P.G.O. Freud James A. Feigenbaum. Discrete scale invariance in stock markets before crashes. Int. J. Mod. Phys., 10:3737-3745, 1996.

[JS98] A. Johansen and D. Sornette. Stock market crashes are outliers. European Physical Journal B, 1:141-143, 1998.

[Lom76] N. R. Lomb. Least-squares frequency analysis of unequally spaced data. Astrophysics and Science, 39:447-462, 1976.

[Mui82] Robb J. Muirhead. Aspects of multivariate statistical theory. Wiley series in probability and mathematical statitstics. John Wiley \& Sons, 1982.

[Sca82] Jeffrey.D. Scargle. Studies in astronomical time series analysis. II. Statistical aspects of spectral analysis of unevenly spaced data. Astrophysical Journal, 263:835-853, 1982.

[SJ01] D. Sornette and A. Johansen. Significance of log-periodic precursors to financial crashes. Quantitative Finance, 1(4):452-471, 2001.

[SLJ99] D. Sornette, O. Ledoit, and A. Johansen. Critical crashes. Risk Magazine, 12(1):91-95, 1999.

[SZ02] D. Sornette and W.-X. Zhou. The US 2000-2002 market descent: How much longer and deeper? Quantitative Finance, 2(6):468-481, 2002.

[VBMA98] N. Vandewalle, Ph. Boveroux, A. Minguet, and M. Ausloos. The krach of october 1987 seen as a phase transition: amplitude and universality. Physica A, 255(1-2):201-210, 1998.

[ZS02] Wei-Xing Zhou and Didier Sornette. Statistical significance of periodicity and log-periodicity with heavy-tailed correlated noise. Int. J. Mod. Phys. C, 13(2):137-170, 2002.

[ZS03] Wei-Xing Zhou and Didier Sornette. Renormalization group analysis of the 2000-2002 anti-bubble in the US S\&P 500 index: Explanation of the hierarchy of 5 crashes and prediction. Physica A, 2003. 


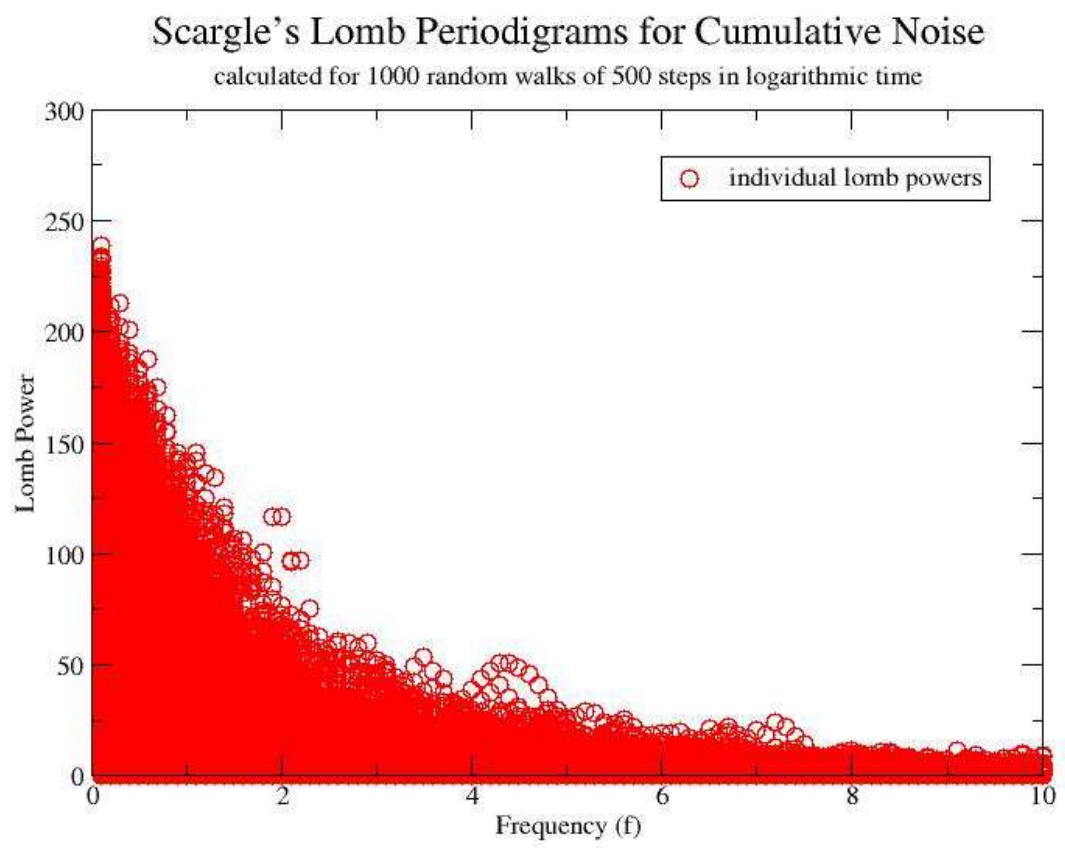

FiguRE 1. Scargle's periodogram for 1000 random walks with normal distributed innovations of zero mean and unit variance. Each random walk has 500 steps which are assumed to occur at times $\log (1) \ldots \log (500)$ 


\section{Cumulative Lomb Periodograms for Cumulative Noise} calculated for 1000 random walks with 500 steps in logarithmic time

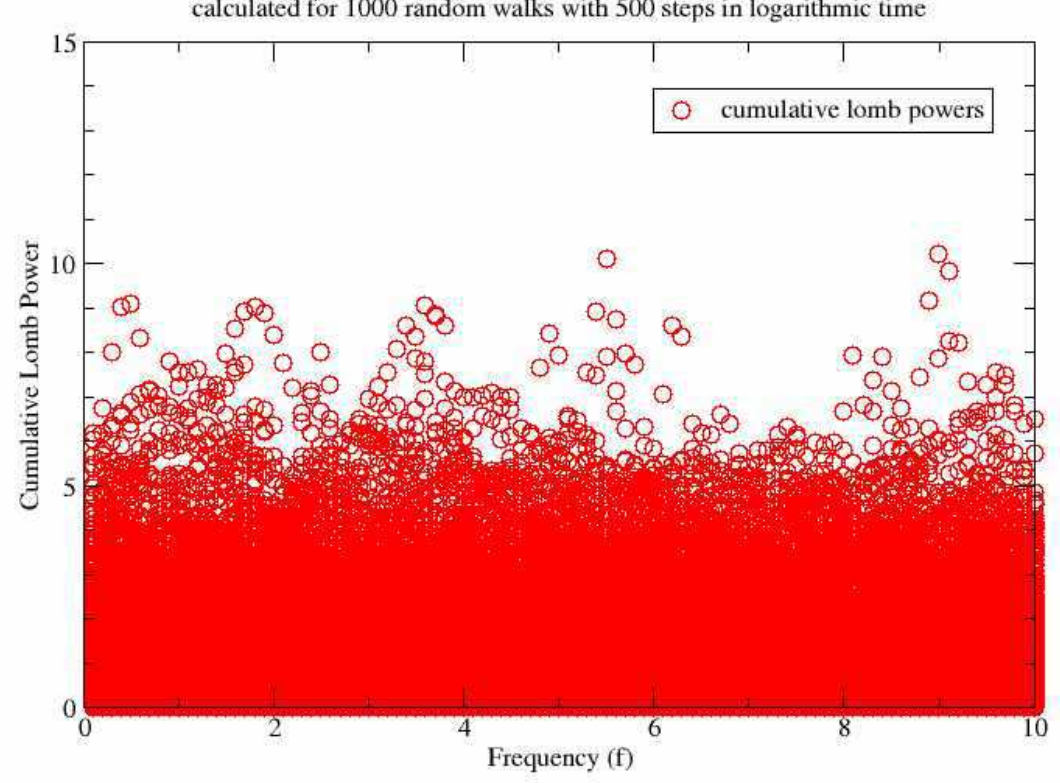

Figure 2. Cumulative Lomb periodogram for 1000 random walks with innovations of zero mean and unit variance. Each random walk has 500 steps which are assumed to occur at times $\log (1) \ldots \log (500)$. For the normalisation $\sigma_{0}=1$ has been used. 
Cumulative Lomb Periodigrams without Correlation Correction

calculated for 1000 random walks with 500 steps in logarithmic time

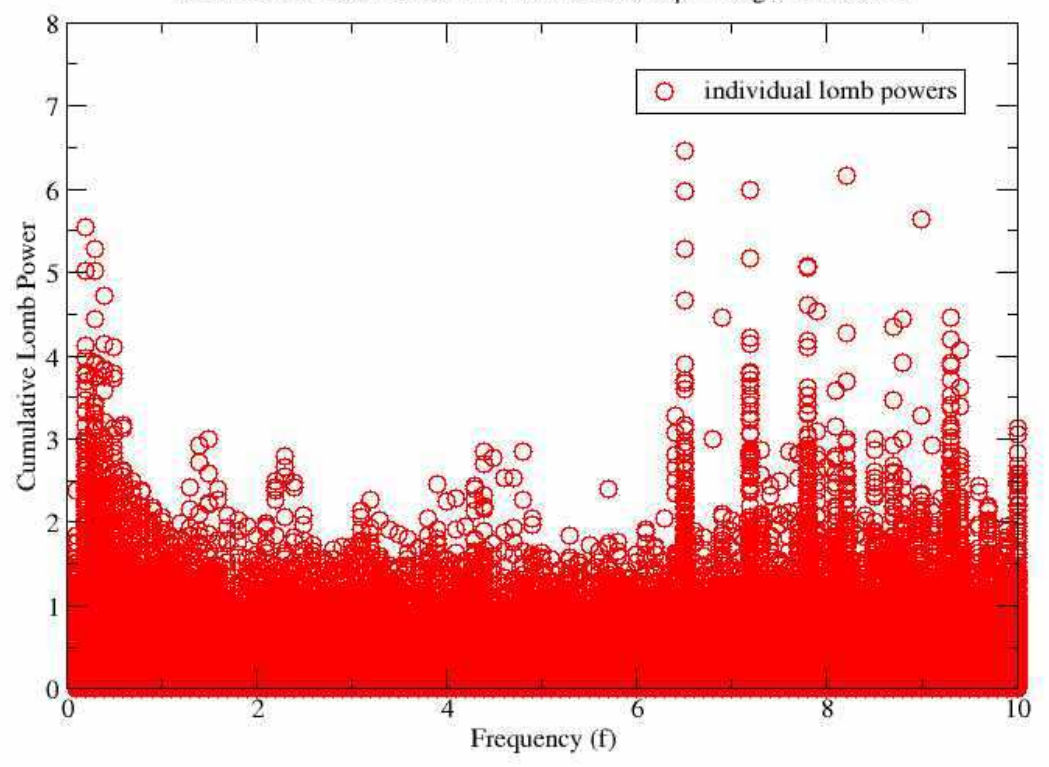

Figure 3. Cumulative Lomb periodograms without the correction for correlation between $C(\omega)$ and $S(\omega)$ of 1000 random walks with innovations of zero mean and unit variance. Each of the random walks has 500 steps which are assumed to occur at times $\log (1) \ldots \log (500)$. $\sigma_{0}$ was estimated for each dataset. The ommission of the correction terms introduces spurious peaks at several frequencies. 
The S\&P 500 Anti Bubble

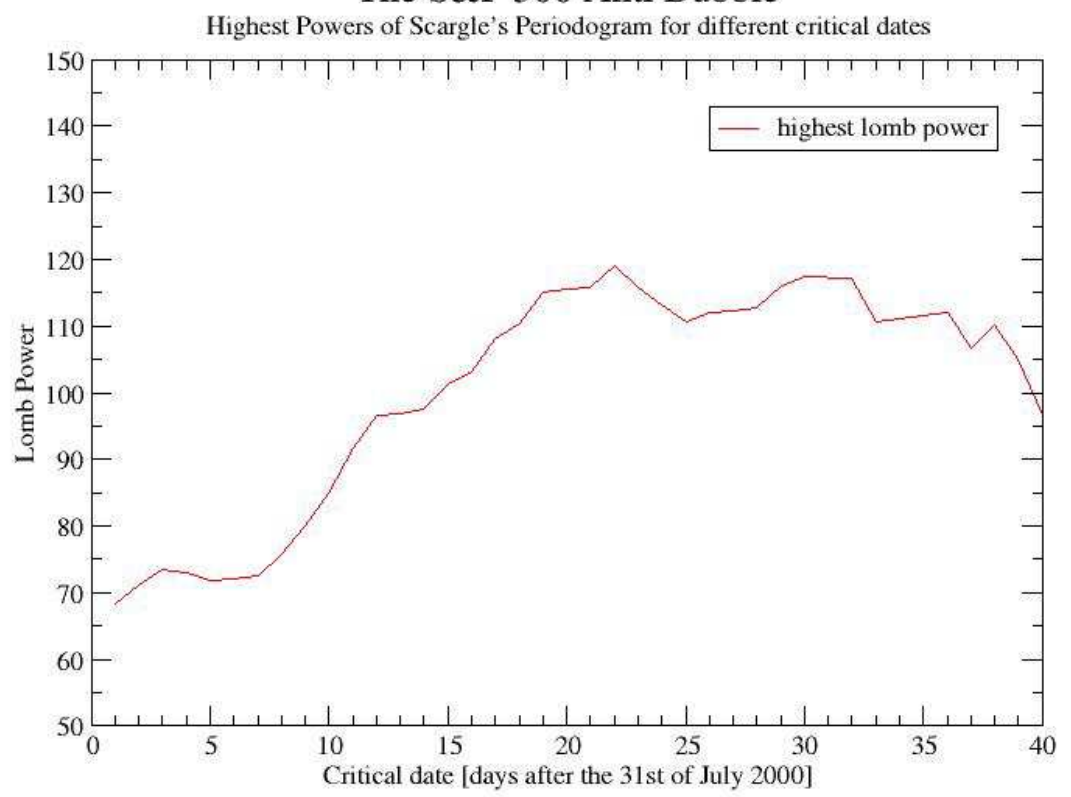

FiguRE 4. Highest peaks in Scargle's periodograms of 2-year windows of S\&P 500 data starting from different days in August and September 2000. Before calculating the periodogram the price data has been detrended according to the procedure described in the text. 


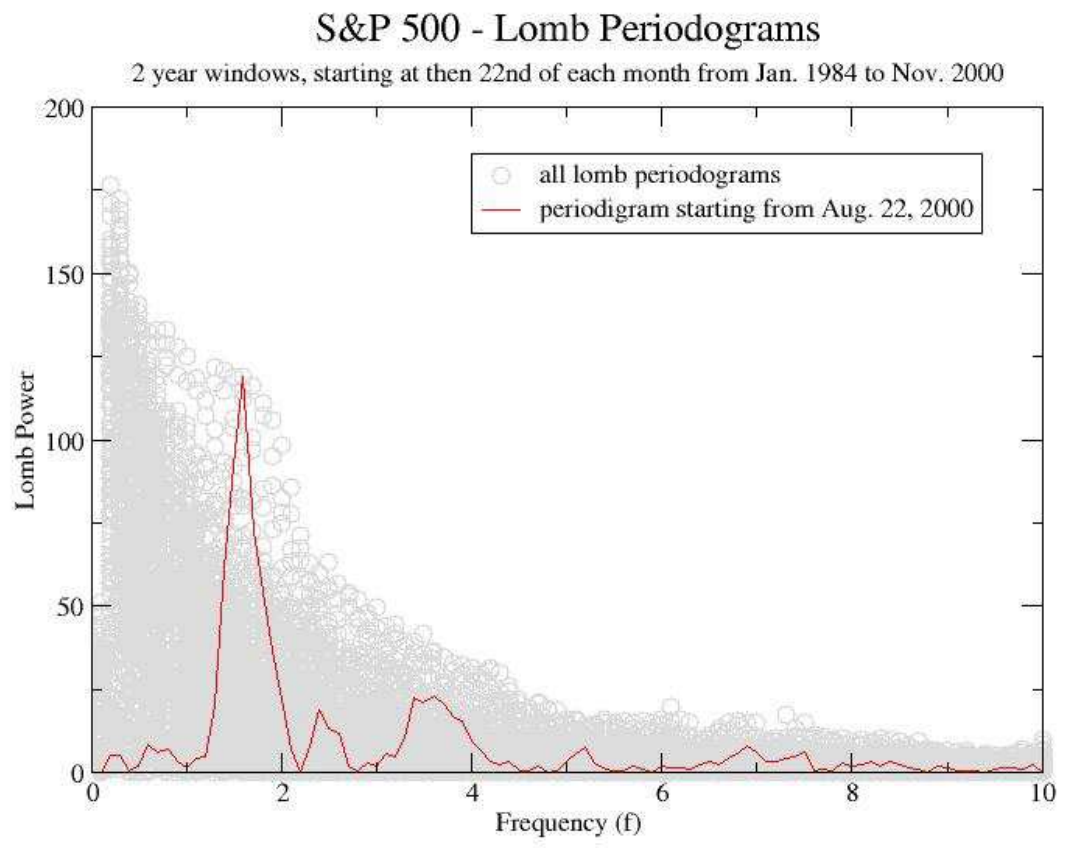

Figure 5. 202 Scargle periodograms for 2-year windows of S\&P 500 data starting from 22nd of each month. Before calculating the periodogram the price data has been detrended according to the procedure described in the text. For one window the detrending procedure did not converge 
Cumulative Lomb Periodigrams of the S\&P 500

2 year windows starting on the 22nd of each month from 1984/01 to 2000/11

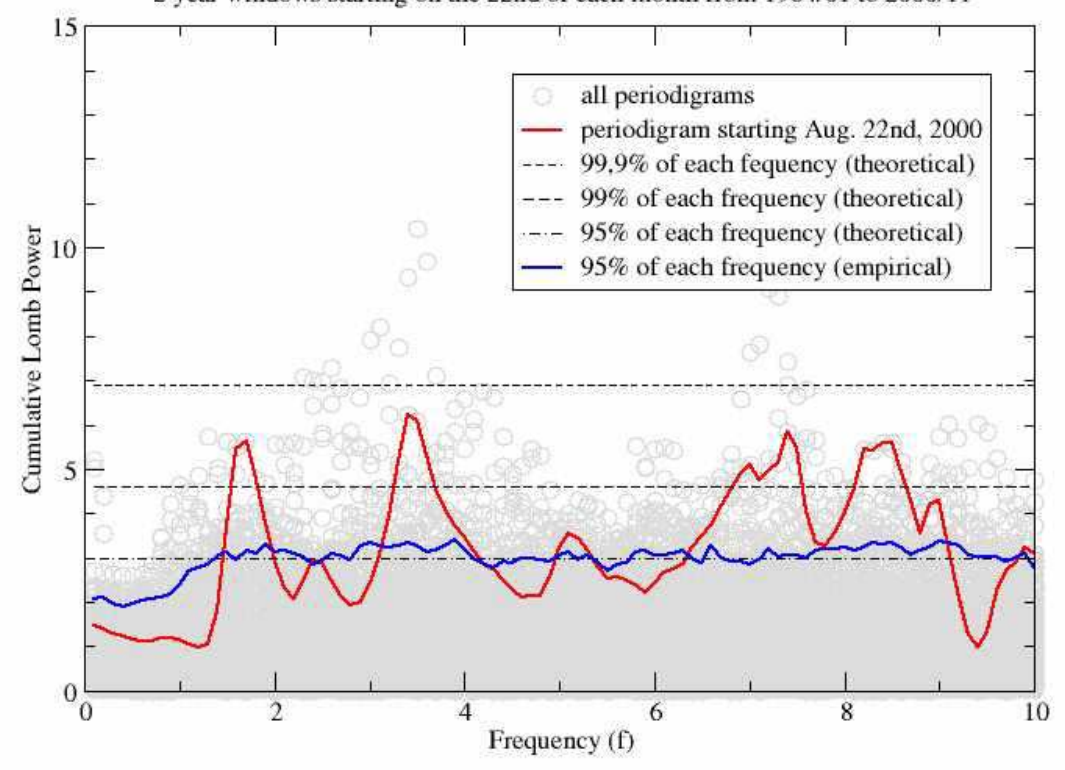

Figure 6. 203 cumulative periodograms for 2-year windows of S\&P 500 data starting from 22nd of each month. The price data has not been detrended. $\sigma_{0}^{2}=0.000119403$ has been estimated from the total 18 years of data. 
Global significance of cumulative lomb powers



FiguRE 7. Highest peaks of cumulative periodograms for 1000 random walks compared with those of 203 cumulative periodograms for 2-year windows of S\&P 500 data starting from 22nd of each month. The price data has not been detrended. $\sigma_{0}^{2}=0.000119403$ has been estimated from the total 18 years of data.

Institut für Mathematik (C), Universität Hannover, Welfengarten 1, D30167 HANNOVER

E-mail address: bothmer@math.uni-hannover.de 